\title{
Proposta de Modelo Socioafetivo de Aluno para a Recomendação de Estratégias Pedagógicas
}

\author{
Carla Adriana Barvinski, Ana Carolina Ribeiro Ribeiro, Magalí Teresinha \\ Longhi, Patricia Alejandra Behar
}

Núcleo de Tecnologia Digital aplicada à Educação (NUTED) - Universidade Federal do Rio Grande do Sul (UFRGS)

Av. Paulo Gama, 110 - Prédio 12105 - 90490-900 - Porto Alegre - RS - Brasil

\{carlabarvinski, carolribeiro2, magali.longhi\}@gmail.com,

pbehareterra.com.br

\begin{abstract}
This article presents a proposal of a socio-affective model of the student as one of the elements for the definition of his profile in a system of recommendation of pedagogical strategies. For that, a reassessment of the current affective model of the student is made, a basic element of the Affective Map, a tool available in the virtual learning environment ROODA. At same time, it is need to incorporate into the model some categories of social information inferred by the Social Map. It is understood that socio-affective strategies can help the teacher to improve his/her pedagogical practice, favoring actions that integrate the student distanced or disinterested.
\end{abstract}

Resumo. Este artigo apresenta uma proposta de modelo socioafetivo do aluno como um dos elementos para a definição do seu perfil em um sistema de recomendação de estratégias pedagógicas. Para tanto, é feita uma reavaliação do atual modelo afetivo do aluno, elemento base do Mapa Afetivo, ferramenta disponibilizada no ambiente virtual de aprendizagem ROODA. Da mesma forma, identifica-se a necessidade de incorporar ao modelo algumas categorias de informações sociais inferidas pelo Mapa Social. Entende-se que as estratégias socioafetivas podem auxiliar o professor a aprimorar sua prática pedagógica, favorecendo ações que integrem o aluno distanciado ou desinteressado.

\section{Introdução}

Este artigo trata do modelo socioafetivo do aluno, elemento necessário para a recomendação de estratégias pedagógicas. O modelo socioafetivo é derivado a partir do Mapa Afetivo e Mapa Social, ferramentas disponibilizadas no ambiente virtual de aprendizagem ROODA - Rede cOOperativa De Aprendizagem para a construção do modelo socioafetivo de aluno. Estuda-se o modelo afetivo do aluno (LONGHI, 2011) a fim de incorporar informações sociais focalizando a recomendação de estratégias pedagógicas mais personalizadas.

As estratégias pedagógicas são ações planejadas, utilizadas pelo docente para atingir os objetivos pretendidos na formação de seus alunos. Nesse sentido, as estratégias afetivas são pensadas para auxiliar o professor a reexaminar ou reconsiderar sua prática pedagógica, promovendo ações que integrem o aluno desmotivado. Já as 
estratégias sociais visam fomentar a inclusão ao debate, a participação efetiva no ambiente, identificando possíveis desistências.

$\mathrm{Na}$ educação presencial o professor interage face a face e utiliza recursos pessoais e subjetivos de percepção do estado afetivo de seus alunos. Já no ensino a distância, tais recursos são prejudicados pelo distanciamento e temporalidade, características dessa modalidade, sendo a interação realizada por meio de tecnologias digitais.

Entre os meios mais utilizados para dar suporte às interações na Educação a Distância (EAD) estão os Ambientes Virtuais de Aprendizagem (AVA), caracterizados como recursos para apoiar a aprendizagem, a comunicação e a colaboração. O ambiente ROODA, um dos AVA institucionalizados na Universidade Federal do Rio Grande do Sul, proporciona um espaço de organização de uma disciplina, execução e avaliação, acesso a materiais, trocas de mensagens, envio de tarefas, etc., favorecendo, assim, a interação e a colaboração. Além disso, o ROODA conta com as ferramentas Mapa Afetivo (MA) (LONGHI, 2011) e Mapa Social (MS) (LONGHI et.al, 2014) para identificar aspectos afetivos e sociais de um aluno. O MA foi concebido para inferir o estado de ânimo do aluno (animado, desanimado, satisfeito e insatisfeito) através da análise de sua atuação no ambiente. O processo de inferência do estado de ânimo envolve a coleta de dados em várias ferramentas do AVA. Os dados inferidos, apresentados sob a forma gráfica, subsidiam a recomendação de estratégias pedagógicas afetivas. Já o MS possibilita, a partir das interações dos usuários nas ferramentas de comunicação no ROODA (Fórum e Contatos), a geração de sociogramas que permitem, de forma visual, acompanhar a relações sociais estabelecidas no AVA. Através do sociograma é possível identificar vínculos, influências e preferências que existem na disciplina ou um em grupo.

Nessa perspectiva, uma proposta de modelo socioafetivo do aluno é apresentado para subsidiar a recomendação de estratégias pedagógicas. Para tal propósito, apresentam-se os ajustes e a inclusão de novas variáveis que passam a integrar o modelo afetivo do aluno, assim como a vinculação das estratégias elaboradas por Longaray (2014). Nessa perspectiva, formula-se a proposta de um sistema de recomendação de estratégias pedagógicas a partir do perfil socioafetivo do aluno.

Este artigo está organizado em cinco seções. Na próxima seção são abordadas as estratégias pedagógicas sociais e afetivas. $\mathrm{Na}$ terceira seção discorre-se sobre os modelos afetivo e social do aluno no AVA ROODA. Na quarta, descrevem-se as modificações no Mapa Afetivo direcionadas à recomendação pedagógica e também a metodologia da pesquisa. Por fim, são apresentadas as considerações finais.

\section{Estratégias pedagógicas sociais e afetivas}

No contexto deste trabalho entende-se por estratégias pedagógicas as ações planejadas e/ou empregadas pelo professor em sua prática a fim de atingir os objetivos pretendidos na formação de seus alunos. Nesse sentido, as estratégias afetivas envolvem o reexame ou reconsideração da prática pedagógica, permitindo ao professor implementar ações que integrem o aluno desanimado ou desinteressado. Quando os alunos são identificados como satisfeitos e animados, tais estratégias apontam a importância de acompanhar e destacar iniciativas dos alunos, como também estimular a autonomia dos mesmos em uma disciplina. 
Na EAD, Souza, Sartori e Roesler (2008) salientam que, pelo fato da mediação do processo educativo ocorrer através de um AVA, necessita-se de ferramentas de comunicação síncrona e assíncrona, proporcionando a implementação de estratégias pedagógicas capazes de promover o diálogo e a participação dos alunos. Para isso, é necessário atentar aos tipos de ferramentas disponibilizadas (chats, fóruns, trocas de mensagens etc.), de forma a planejar como elas devem ser utilizadas nos diferentes momentos. Cada ferramenta exige uma série de habilidades diferenciadas e implica no desenvolvimento de estratégias pedagógicas direcionadas a diferentes objetivos, tais como a atenção aos estados de ânimo do aluno e o foco nos aspectos sociais. Segundo Longaray (2014), o uso de diferentes recursos comunicacionais visa o desenvolvimento das potencialidades cognitivas e afetivas do aluno, tanto em âmbito individual quanto coletivo. Porém, ressalta que o reconhecimento dos aspectos afetivos na aprendizagem implica a adoção de uma prática pedagógica que envolva o processo de construção do conhecimento que seja permeado pela afetividade e não apenas pela cognição, conforme destaca Piaget (2014).

De acordo com Longaray, Ribeiro e Behar (2013), na EAD, o tipo da tarefa e a estratégia pedagógica influenciam o fenômeno afetivo manifestado e vivenciado pelo aluno. Para Longaray (2014), é desafiadora a adoção de estratégias que minimizem a ausência do contato presencial do professor em relação ao aluno, do aluno e colegas, bem como a elaboração de práticas que potencializam as relações afetivas. Nesse sentido, o mapeamento de estados de ânimo, propiciado pelo MA revela-se como uma ferramenta adequada para recomendar uma estratégia pedagógica afetiva mais adequada à situação do aluno, visando tanto a recondução do aluno aos estudos ou, ainda, ampliando as possibilidades de aprendizagem.

A partir do MS, o professor pode atender a sugestão de estratégia social indicada pelo recurso de recomendação da ferramenta. Tal recurso leva em consideração o cálculo do grau de uma categoria social, a saber: colaboração, popularidade, agrupamento, distanciamento pela turma, ausência e evasão. $O$ recurso de recomendação realiza uma mineração das interações efetuadas nas ferramentas de comunicação no ROODA (Fórum, Bate-papo, Diário de Bordo, Contatos e Comentários das funcionalidades Webfolio e Biblioteca) para definir o grau da categoria.

As categorias de informações sociais apresentam uma forma de interpretação das relações mais corriqueiras demonstradas pelos mapas avaliados pela equipe pedagógica da pesquisa. Desse modo, contribuem para a identificação do perfil social de um aluno e a indicação de estratégias pedagógicas para aumentar ou diversificar as interações sociais entre os alunos. Tais estratégias pedagógicas são importantes para qualificar o ensino, uma vez que os aspectos sociais de cada indivíduo estão intrinsecamente relacionados com a cognição (PIAGET, 2014), ou seja, estão sempre envolvidos com a aprendizagem.

\section{Modelos Afetivo e Social do aluno no AVA ROODA}

A implementação do mapeamento dos estados de ânimo no AVA ROODA fundamentase na teoria de Piaget (2005) e Scherer (2005). Segundo Longhi (2011), os trabalhos de Scherer e Piaget são importantes para a distinção e compreensão de como e porque os fenômenos afetivos se manifestam, como também a identificação dos estados de ânimo relacionados ao processamento cognitivo da aprendizagem e à reorganização da 
informação na memória (LONGHI, BEHAR e BERCHT, 2009). Para Scherer (2005), a afetividade é descrita por um conjunto de fenômenos afetivos. Como tal, eles se distinguem pela origem, função, intensidade, duração, repercutindo na reação corporal e na motivação para decidir qual ação comportamental tomar. Para diferenciá-los e definilos, o autor classificou-os em sete classes, a saber: emoções utilitárias (ou básicas), emoções estéticas, estados de ânimo, preferências, atitudes, disposições afetivas (ou traços de personalidade) e posturas interpessoais (ou estilos afetivos).

Os estados de ânimo se caracterizam como emoções de segundo plano (DAMÁSIO, 1996), de caráter ondulatório e difuso, de baixa intensidade, de longa duração, que surgem, muitas vezes, de modo desconhecido, podendo ser originados a partir das emoções primárias e secundárias (ROSENBERG, 1998). Mais ainda, eles repercutem na cognição de forma positiva ou negativa por horas ou dias (SCHERER, 2005), afetando tanto a reflexão e a tomada de decisão, quanto o próprio desempenho do aluno. Dentre os estados de ânimo, incidem, com maior ênfase na aprendizagem, os estados de animação e satisfação (Longhi, 2011), na valência positiva e negativa, razão pela qual foram implementados no MA.

Os estados de ânimo estão vinculados aos traços de personalidade e às emoções. Os traços de personalidade sinalizam padrões que caracterizam como um sujeito percebe, sente e atua nas mais variadas situações. Segundo Pacheco e Sisto (2003), o traço de personalidade refere-se à parte constante do comportamento de uma pessoa, já que a personalidade consiste em uma parte variável e outra mais persistente. Ressaltam os autores que os traços "são tendências amplas em mudança contínua", mesmo quando o indivíduo modifica seu comportamento. Aspectos constantes deste são passíveis de reconhecimento por representar produto de vários traços de personalidade. Rosenberg (1998), por fim, esclarece que os traços de personalidade, os estados de ânimo e as emoções constituem uma estrutura hierárquica. Os estados de ânimo são compostos de emoções. Ambos modelam os traços de personalidade, determinados através de modelos caracterizados como fatores (ou dimensões).

Tais conceitos contribuiram para a implementação do framework ROODAAfeto, a estrutura tecnológica que suporta a ferramenta Mapa Afetivo para fins de reconhecimento dos estados de ânimo do aluno. O framework ROODAAFETO incorpora outros dois frameworks e uma máquina de inferência baseada em Redes Bayesianas. Tais componentes permitem o desenho do mapa comportamental-afetivo do aluno em interação no AVA ROODA. Dessa forma, o estado afetivo é visualizado pelo professor através de três gráficos: o da Subjetividade em Texto, o de Fatores Motivacionais e o Geral de Afetividade do aluno. A subjetividade em texto, os fatores motivacionais e os traços de personalidade compõem a máquina de inferência do provável estado de ânimo do aluno.

A inferência da subjetividade afetiva é feita pelo framework AWM (LONGHI, BEHAR \& BERCHT, 2011), que identifica e classifica as palavras de conotação afetiva existentes em um texto. Há várias formas de detecção de emoções na interação humanocomputador conforme apresenta Cowie et. al (2001). Podem ser identificadas através da fala (POLZIN \& WAIBEL, 1998), das expressões faciais (DE SILVA, MIYASATO \& NAKATSU, 1997), alterações do estado fisiológico (KIM, BANG \& KIM, 2004; HAAG et.al, 2004) e produção textual (WU, CHUANG \& LIN, 2006). Todavia, na ausência de contato face a face para detectar expressões faciais e entonações na voz, a 
VI Congresso Brasileiro de Informática na Educação (CBIE 2017)

Anais do XXVIII Simpósio Brasileiro de Informática na Educação (SBIE 2017)

alternativa é decifrar as emoções do texto nos fóruns on-line (BINALI, WU \& POTDAR, 2010). A detecção de emoção em texto objetiva inferir as emoções subjacentes que influenciam seu autor. Baseia-se na premissa de que, se uma pessoa está feliz, isso a influenciará a se expressar textualmente, usando palavras positivas. Do mesmo modo, se a pessoa estiver triste, frustrada ou irritada, as palavras que utiliza denotará emoções negativas (BINALI, WU \& POTDAR, 2010).

O processo adotado no AWM consiste em minerar o texto para extrair os lexemas afetivos. Posteriormente, eles são classificados e na sequência, determinada sua posição dentro da Roda dos Estados Afetivos (LONGHI, 2011), sinalizando, então, o estado de ânimo, conforme ilustra a Figura 1. No sentido horário, o primeiro quadrante da Roda dos Estados Afetivos indica o estado de ânimo Safistação; o segundo, Animação; o terceiro, Desânimo; e o quarto, Insatisfação.

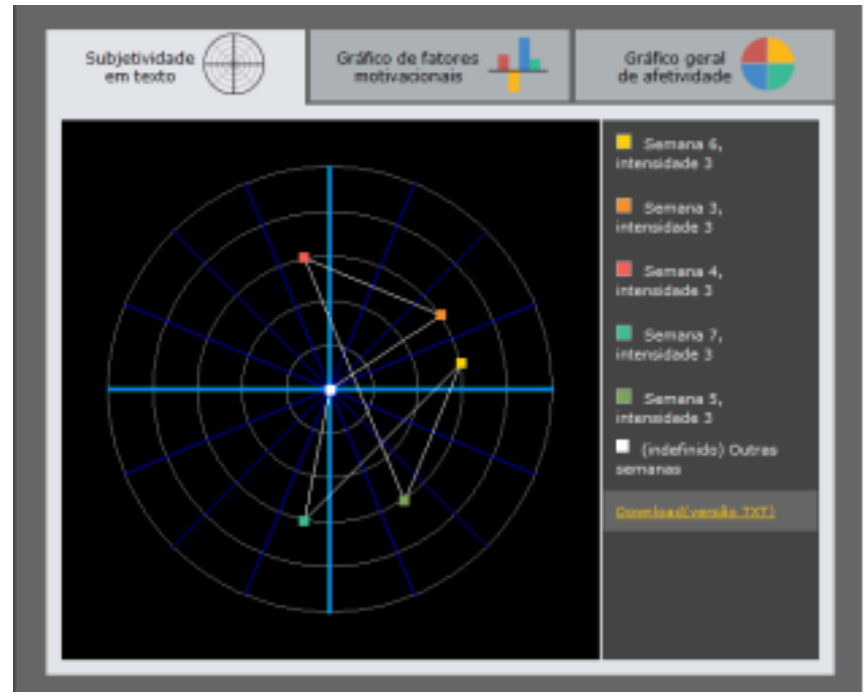

Figura 1: Gráfico de Subjetividade em Texto. Fonte: LONGHI, 2011.

Segundo Longhi, Behar e Bercht (2011), o conjunto sistemático de ações adotadas pelo aluno no AVA representa o comportamento observável. A identificação dos padrões comportamentais do aluno, expressam sua motivação ou seu "grau motivacional" e é um indicador dos estados de ânimo. Nesse enfoque, o MA considera os fatores motivacionais Confiança, Esforço e Independência (BERCHT, 2001) para inferência do grau motivacional, proporcionada pelo framework BFC (Behavioral Factor Calculation) descrito em LONGHI et.al (2010). Para o cálculo, são consideradas sete variáveis relacionadas ao comportamento observável do aluno, às quais são atribuídas ponderações (BERCHT, 2001). Essas variáveis representam dados qualitativos e quantitativos referentes ao comportamento nas ferramentas Fórum, Batepapo, Diário de Bordo e Contatos. O cálculo de cada fator motivacional considera o conjunto dessas ferramentas.

O traço de personalidade do aluno constitui o terceiro aspecto considerado no MA. A especificidade da personalidade é inferida mediante questionários ou inventários, sob a forma de testes psicométricos que avaliam os traços ou facetas. Vários são os inventários para traços de personalidade. Longhi (2011) optou pelo Inventário Fatorial da Personalidade (IFP) (PASQUALI, AZEVEDO e GHESTI, 1997), 
cuja aplicação e análise foi conduzida por profissional credenciado. A análise dos questionários de traços de personalidade, realizada por psicólogo, foi processada digitalmente de modo a compor uma base de dados psicométricos. Posteriormente, o conjunto das informações afetivas de cada aluno coletadas pelos frameworks BFC e AWM, juntamente com os dados de traços de personalidade extraídos do questionário IFP, foram submetidos a uma Rede Bayesiana (RB), compondo o Modelo Afetivo do aluno. A inferência resultante revela o provável estado de ânimo. Destaca-se que uma Rede Bayesiana se caracteriza por ser um tipo de rede semântica representada por um grafo orientado acíclico, em que cada nodo (ou nó) é identificado com informações de probabilidade (RUSSEL e NORVING, 2004). Ela pode ser analisada pelo viés qualitativo e quantitativo, o que, segundo Longhi, Behar e Bercht (2010) justifica sua adoção.

Assim, o modelo afetivo do aluno é composto pelas variáveis de evidências: traços de personalidade, subjetividade em texto e fatores motivacionais (confiança, esforço e independência). Tais variáveis definem a expectativa de um estado de ânimo ocorrer em um determinado período de tempo. O estado de ânimo de maior probabilidade serve de elemento para a escolha da sugestão de estratégia pedagógica afetiva.

Por outro lado, a construção de sociogramas pelo MS está baseada nas ideias de Moreno (1972). O objetivo da sociometria consiste no estudo matemático das propriedades psicossociológicas das populações, de forma a compreender a estrutura de um grupo. Uma das técnicas da Sociometria é a aplicação de testes sociométricos que permitem visualizar as semelhanças e as diferenças entre os indivíduos que compõem um grupo. Contudo, no MS são buscadas informações na forma de interação do aluno com outros participantes (por exemplo, se enviou mensagem apenas para professor, apenas para determinados colegas ou para toda turma).

Os sociogramas explicitam visualmente a posição ocupada pelo indivíduo no grupo e o núcleo de relações que se formam ao seu redor. Esse núcleo de relações constitui a menor estrutura social, que Moreno define como átomo social (MORENO, 1972). Enquanto certos átomos sociais limitam-se aos indivíduos que participam nele, parte desses indivíduos podem se relacionar com partes de outros átomos sociais, assim sucessivamente, formando cadeias complexas de inter-relações. Então, através de um sociograma pode-se perceber a posição social de cada participante de uma comunidade de aprendizagem e sua relação com o restante do grupo. Através das escolhas emitidas determina-se quem do átomo social é o mais privilegiado e os que exercem a reciprocidade; quais indivíduos são os rejeitados por não cumprir a reciprocidade; e quais são os isolados por não emitirem preferências.

O modelo social do aluno é composto pela variável posição social, a partir do qual é verificado o grau de colaboração, popularidade, agrupamento, distanciamento pela turma, ausência e evasão, definidas como categorias de informação social.

\section{Viabilizando a recomendação de estratégias pedagógicas}

Dos três gráficos disponibilizados pelo MA, apenas o da Subjetividade Afetiva em Texto é contemplado com estratégias pedagógicas. Os demais estão sendo explorados para a composição de novas estratégias. Já o MS apresenta estratégias em sentido geral 
VI Congresso Brasileiro de Informática na Educação (CBIE 2017)

Anais do XXVIII Simpósio Brasileiro de Informática na Educação (SBIE 2017)

por categoria de informação social no momento da exibição, não levando em conta a história de interações ou outras categorias que eventualmente o aluno se insira.

Nesse aspecto, para dotar o AVA ROODA de um sistema computacional que, a partir do MA e MS, sugira estratégias pedagógicas personalizadas capazes de atender às demandas socioafetivas dos alunos, há a necessidade de ajustes importantes na ferramenta MA. Cabe destacar, a substituição do inventário IFP pelo Big Five. Tal decisão deve-se à possibilidade de obter os traços de personalidade do aluno online, a partir do Portal Personalitatem ${ }^{1}$, desenvolvido na Universidade Federal de Sergipe. Ao mesmo tempo, novas variáveis estão sendo inseridas no cálculo dos fatores motivacionais, em função da renovação das funcionalidades e/ou recursos do AVA ROODA. Com isso, acredita-se seja possível derivar estratégias pedagógicas afetivas mais personificadas.

A junção dos modelos afetivo e social definirá o modelo socioafetivo do aluno, ilustrado na Figura 2, na medida em que contempla a representação dos processos, variáveis e relacionamentos dos aspectos socioafetivos em AVA. Tal modelo contribui para a definição do perfil do aluno, colaborando para o reconhecimento dos interesses e das preferências para o aprendizado.

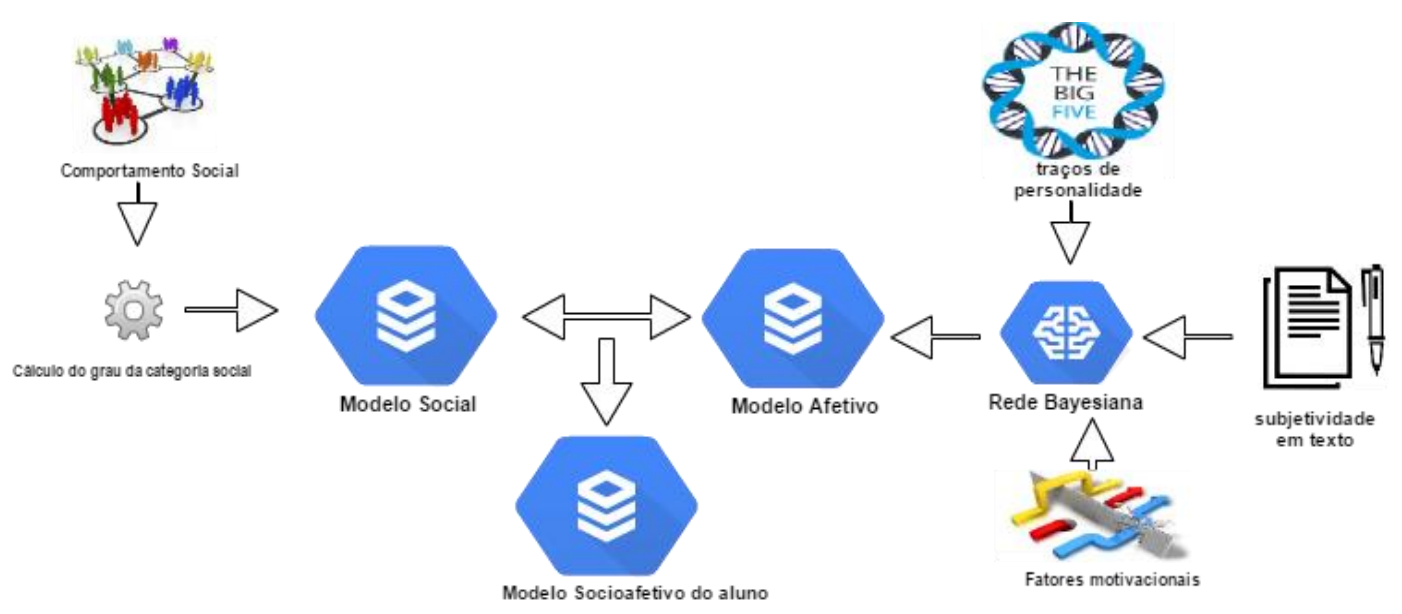

Figura 2: Modelo Socioafetivo do aluno.

Acredita-se que a definição mais próxima do perfil real do aluno (incluindo-se o perfil socioafetivo) favorece a recomendação de estratégias pedagógicas mais pertinentes ao contexto, sem desconsiderar um estreitamento de laços sociosafetivos na EAD. Pretende-se, desse modo, contribuir para minimizar a evasão escolar.

A Figura 3 apresenta a arquitetura do Sistema de Recomendação de Estratégias Pedagógicas baseadas no perfil socioafetivo do aluno. O Sistema Recomendador recebe as informações afetivas e sociais, respectivamente do Mapa Afetivo e Mapa Social, construindo o perfil socioafetivo do aluno. Este perfil é utilizado como uma das informações para o processo de filtragem. Na sequência é escolhida a estratégia socioafetiva mais personalizada.

${ }^{1}$ Disponível em: https://personalitatem.ufs.br/inventory/ 


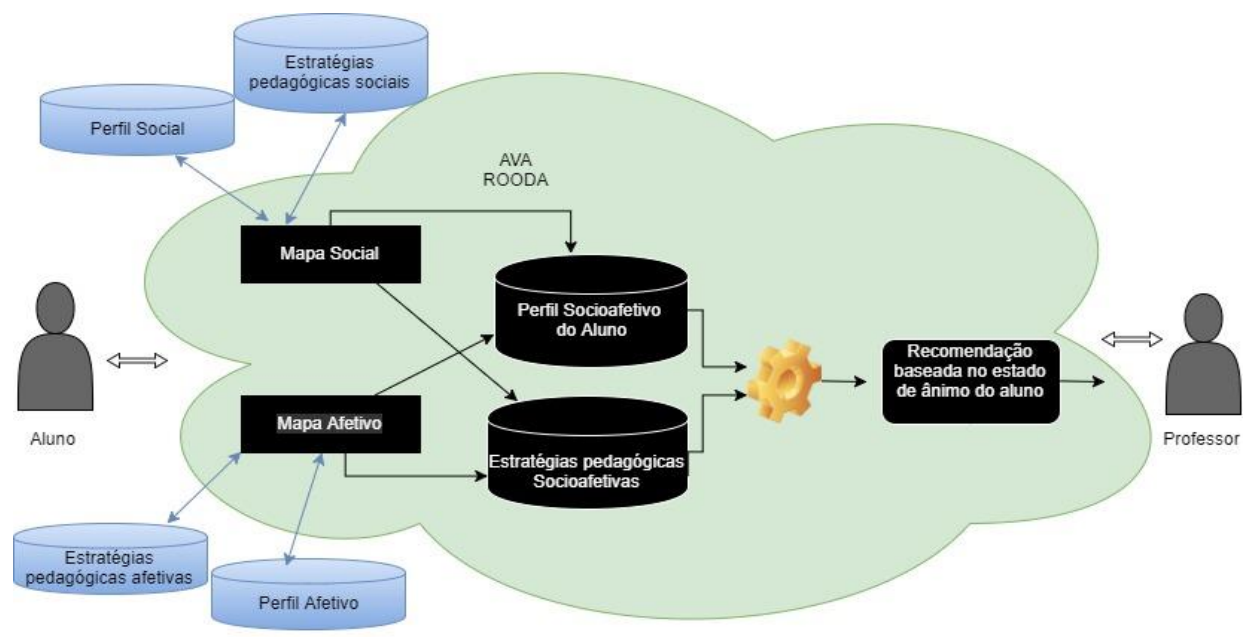

Figura 3: Arquitetura do Sistema de Recomendação Pedagógica.

A metodologia para validação do protótipo consta de uma análise qualiquantitativa, cujos dados socioafetivos de cada aluno estão sendo coletados junto aos Mapas Afetivo e Social de disciplinas já concluídas. A análise de dados quantitativo está sendo organizada de modo a ser aplicada técnica de Learning Machine (a exemplo WEKA). Quanto aos dados qualitativos, a equipe pedagógica analisa os textos das funcionalidades Fórum e Diário de Bordo e os sociogramas extraídos do MS. Assim que o recomendador estiver implementado, avalia-se as estratégias pedagógicas sugeridas. Tão logo os ajustes e correções forem implementados, o recurso de recomendação das estratégias pedagógicas socioafetivas será aplicado para validação em atividades de ensino em nível de graduação, pós-graduação e/ou extensão, com a construção de instrumentos de comparação.

\section{Conclusões}

O MA é uma funcionalidade do AVA ROODA que infere o estado de ânimo do aluno em determinado período. A inferência de aspectos afetivos a partir de produções textuais ensejou a elaboração de estratégias pedagógicas afetivas, sugeridas ao professor no Gráfico de Subjetividade em Texto. Os demais recursos da funcionalidade, quais sejam o Gráfico de Fatores Motivacionais e o Mapa Geral de Afetividade, também viabilizam a elaboração de novas estratégias pedagógicas que consideram o estado de motivação do aluno e seus traços de personalidade.

O MS é a ferramenta do AVA ROODA que define a posição social de um aluno a partir do cálculo de cinco categorias: colaboração, popularidade, agrupamento, distanciamento pela turma, ausência e evasão.

Estão em curso aprimoramentos no MA, com o intuito de melhorar a inferência dos aspectos afetivos. Por outro lado, os aspectos sociais oriundos do MS estão sendo incorporados no Modelo de Aluno de modo a constituir o Modelo SocioAfetivo do Aluno. O processo de inferência do estado de ânimo no MA em conjunto com o perfil social pode subsidiar o desenvolvimento de estratégias pedagógicas mais condizentes com as necessidades e interesses do aluno. Uma vez consolidado o modelo socioafetivo, serão derivadas as estratégias pedagógicas possíveis de implementação no sistema de recomendação em futuro desdobramento da presente pesquisa. 
VI Congresso Brasileiro de Informática na Educação (CBIE 2017)

Anais do XXVIII Simpósio Brasileiro de Informática na Educação (SBIE 2017)

\section{Referências}

Behar, P. A.; Ribeiro, A. C. R.; Schneider, D.; Silva, K. K. A.; Machado, L. R.; Longhi, M. T., (2013) "Educação a distância e competências: uma articulação necessária", In: Competências em Educação a Distância, Org.: Patricia Alejandra Behar, 1ed. Porto Alegre: Penso, v. 1, p. 35-50.

Bercht, M., (2001) "Em direção a agentes pedagógicos com dimensões afetivas", Tese Doutorado, Universidade Federal do Rio Grande do Sul, Programa de Pós Graduação em Computação.

Binali, H.; Wu, C.; Potdar, V., (2010). "Computational approaches for emotion detection in text", In: IEEE 8th International Symposium on Intelligent Systems and Informatics, IEEE, p. 172-177.

Cowie R., Cowie D., Tsapatsoulis N.; Votsis G., Kollias S., (2001) "Emotion recognition in human-computer interaction," In: IEEE Signal Processing Magazine, vol. 18(1).

Damásio, A. R., (1996), O erro de Descartes: emoção, razão e cérebro humano, Companhia das Letras.

De Silva, L.C.; Miyasato, T.; Nakatsu, R., (1997) "Facial emotion recognition using multi-modal information", In: Information, Communications and Signal Processing, ICICS, Proceedings of 1997 International Conference on IEEE, p. 397-401.

Haag, A.; Goronz, S; Schaich, P.; and Williams, J., (2004) "Emotion Recognition Using Bio-sensors: First Steps towards an Automatic System", In: Affective Dialogue Systems, Springer Berlin/Heidelberg, vol. 3068.

Kim, K. H.; Bang, S. W.; and Kim, S. R., (2004) "Emotion recognition system using short-term monitoring of physiological signals ", In: Medical and Biological Engineering and Computing, vol. 42, p. 419-427.

Longaray, A. N. C.; Ribeiro, A. C. R.; Behar, P. A., (2013) "Estratégias Pedagógicas para a Educação a Distância: um olhar a partir dos estados de ânimo do aluno", In: Congreso Internacional de Informática Educativa - TISE. Vol. 9.

Longaray, A. N. C., (2014) "Estratégias para a educação a distância: um olhar a partir dos estados de ânimo do aluno". Dissertação de Mestrado. Universidade Federal do Rio Grande do Sul. Programa de Pós-Graduação em Educação.

Longhi, M. T., (2011) "Mapeamento de aspectos afetivos em um ambiente virtual de aprendizagem". Tese de doutorado. Universidade Federal do Rio Grande do Sul. Programa de Pós-Graduação em Informática na Educação.

Longhi, M. T., Behar, P.A., and Bercht, M., (2011) "Inferência dos estados de ânimo do aluno em um ambiente virtual de aprendizagem baseada em redes bayesianas", Informática na educação: teoria \& prática, v.14, n.1.

Longhi, M. T., Behar, P.A., and Bercht, M., (2009) "O sujeito afetivo e os ambientes virtuais de aprendizagem”, RENOTE, v. 7, n. 3, p. 275-285.

Longhi, M. T., Behar, P.A., and Bercht, M., (2010) "Máquina de inferZência baseada na 
VI Congresso Brasileiro de Informática na Educação (CBIE 2017)

Anais do XXVIII Simpósio Brasileiro de Informática na Educação (SBIE 2017)

Longhi, M. T., Behar, P.A., and Bercht, M. and Simonato, G., (2010) "Os fatores motivacionais e os estados de ânimo em ambientes virtuais de aprendizagem", In: Congresso Iberoamericano de Informática Educativa. Chile, p. 551-558.

Longhi, M. T; Machado, L. R.; Ribeiro, A. C. R.; Behar, P. A., (2014) "Mapa social: ferramenta sociométrica para mapear as interações sociais na educação a distância", In: Congreso Internacional sobre Aplicación de Tecnologías de la Información y Comunicaciones Avanzadas.

Moreno, J.L., (1972) "Fundamentos de la Sociometria", Paidós.

Pacheco, L.; Sisto, F. F., (2003) "Aprendizagem por interação e traços de personalidade", Psicologia Escolar e Educacional, v. 7, n. 1, p. 69-76.

Pasquali, L.; Azevedo, M. M.; Ghesti, I., (1997) "Inventário Fatorial de Personalidade: manual técnico e de aplicação", Casa do Psicólogo.

Piaget, J., (2005) "Inteligencia Y afectividad”, Editora AIQUE.

Piaget, J., (2014) "Relações entre a Afetividade e a Inteligência no Desenvolvimento Mental da Criança", Wak Editora.

Polzin, T. S. and Waibel, A. H., (1998) "Detecting Emotions in Speech," In: Proceedings of the CMC.

Rosenberg, E. L., (1998), "Levels of analysis and the organization of affect", Review of General Psychology, v. 2, n. 3, p. 247-270.

Russel, S.J.; Norving, P., (2004) "Inteligência Artificial”, Elsevier.

Scherer, K. R., (2005) "What are emotions? And how can they be measured?", In: Social Science Information, 44 (4), pp. 695-729.

Souza, A. R. B; Sartori, A. S.; Roesler, J., (2008) "Mediação Pedagógica na Educação a Distância: entre enunciados teóricos e práticas construídas”, Rev. Diálogo Educ., v. 8, n. 24, p. 327-339.

Wu, C.; Chuang, Z.; Lin, Y., (2006) "Emotion recognition from text using semantic labels and separable mixture models", In: ACM transactions on Asian language information processing (TALIP), v. 5, n. 2, p. 165-183. 